\title{
Eulerian models of the rotating flexible wheelset for high frequency railway dynamics
}

\author{
Authors: Luis Baeza ${ }^{1 *}$, Juan Giner-Navarro ${ }^{1}$, David J. Thompson ${ }^{2}$, Juan Monterde ${ }^{3}$ \\ ${ }^{1}$ Centro de Investigación en Ingeniería Mecánica, Universitat Politècnica de València, Valencia, Spain. \\ ${ }^{2}$ Institute of Sound and Vibration Research, University of Southampton, Southampton, UK. \\ ${ }^{3}$ Departamento de Matemáticas, Universitat de València, Burjassot, Spain.
}

\section{* E-mail corresponding author: baeza@mcm.upv.es}

\begin{abstract}
In this paper three formulations based on an Eulerian approach are presented to obtain the dynamic response of an elastic solid of revolution, which rotates around its main axis at constant angular velocity. The formulations are especially suitable for the study of the interaction of a solid with a nonrotating structure, such as occurs in the coupled dynamics of a railway wheelset with the track. With respect to previous publications that may adopt similar hypotheses, this paper proposes more compact formulations and eliminates certain numerical problems associated with the presence of second-order derivatives with respect to the spatial coordinates. Three different models are developed depending the basis function that represents the displacements associated with the deformation; these basis functions are: (1) the shape functions that are used in the three dimensional finite element (FE) method; (2) the undamped mode shapes of the solid; (3) the shape functions that are adopted in the axisymmetric FE approach. Comparisons are shown of calculations carried out using these models. These show the existence of modal veering when analysing the Campbell diagram for a railway wheel.
\end{abstract}

\section{Keywords}

Vehicle-track interaction, rotating solid, Eulerian coordinates, rotors, mode veering. 


\section{INTRODUCTION}

Wheels, shafts, brake discs and many other rotating solids are widely used in all fields of industry. Consequently, there has been considerable research effort to include the inertial effects associated with the rotation in computationally efficient models. Simplified formulations from the beginning of the last century, in particular Jeffcott rotors (zero-dimensional domain through lumped parameters) and rotating Rayleigh beams (one-dimensional), have been widely adopted in the literature. These show the frequency shift of the resonance peaks of the rotating structure with the angular velocity (see monographs in [1,2]). Whereas one- and two-dimensional models can be studied by means of a non-rotating frame of reference, the leap to three-dimensional solids with arbitrary geometry would require a reference frame that rotates with the solid (Lagrangian approach). This method can be followed by describing small displacements with respect to a non-deformed configuration (see Refs. $[3,4]$ ) or adopting the Finite Element (FE) method, an option that has been consolidated due to the rapid growth of computational performance in the last few decades $[5,6]$.

Nevertheless, Lagrangian coordinates are not particularly suitable for rotating structures when interacting with non-rotating ones, where the interaction force is applied at a fixed spatial point; there is thus a relative motion between the load and the rotating solid, hence requiring frequent remeshing operations and leading to high computational effort and numerical issues. This has motivated researchers in recent years to investigate techniques to adapt the FE approach to more numerically efficient models.

In Ref. [7], nonlinear Arbitrary Lagrangian-Eulerian (ALE) coordinates [8] were implemented for solids in rolling contact, allowing large distortions in rotating tyres to be handled with a modified FE formulation; this describes their stationary motion in a fixed-reference frame through which the rotating material particles flow. This method is characterised by the use of an arbitrary reference configuration which is neither fixed in space nor connected with the material particles; the boundaries of the domain are moving like the material boundaries. This formulation was extended to modal coordinates (Modal-ALE approach, see Ref. [9]) in the low frequency range by means of solving the eigen-problem from the FE code and applying the modal coordinate transformation to account for the tyre rotation.

For rotating solids undergoing small distortions (e.g. railway wheelsets which are much stiffer than tyres), Ref. [10] proposed an Eulerian approach initially based on Shabana's formulation [4] by using 
a non-rotating reference frame. Using this model it is possible to take advantage of the properties of the solids of revolution since their mode shapes in a fixed coordinate frame do not depend on the rotation of the solid; they can hence be used as basis functions to define a generic displacement of any spatial point of the rotating solid, leading to great reductions of the computational cost. Following this methodology, Eulerian-modal coordinates were introduced in Ref. [11] by adopting the mode shapes of the non-rotating solid as basis functions. The formulation of the models published in Refs. $[10,11]$, both of which adopt Cartesian coordinates, includes a volumetric integral term that contains a second-order derivative. This leads to a numerical problem since the discretisation of the domain of a three-dimensional solid (i.e. the FE discretisation) is not able to guarantee the $\mathrm{C}^{1}$-continuity of functions (i.e. continuity of their first derivatives) between its subdomain edges. Therefore, it cannot be guaranteed that the second-order derivative is bounded (see Ref. [12]); the associated integral term can also diverge or give greater numerical errors when the mesh is refined.

The present work describes a methodology to study the dynamics of solids of revolution that is based on an Eulerian approach. The paper presents three different models described by a closed-form formulation in cylindrical coordinates in which the small displacements due to the deformations are expressed by means of separation of variables: a set of basis functions that depends on space, and the corresponding generalised coordinates that depend on time. This general formulation can be found in section 2.1 of the present work; it is more compact than that published in Refs. [10,11] and solves the numerical problem associated with the presence of second-order derivatives.

The general formulation of the rotating solid is then adopted in order to obtain equations of motion (EOM) for the rotating solid when the basis functions are: (i) the shape functions of the FE method (section 2.2); (ii) the undamped mode shapes (section 2.3); or (iii) based on the axisymmetric FE approach (section 2.4). All three formulations are especially useful to assess the dynamics of railway wheels and wheelsets, and the results presented in section 3 focus on studies performed for this type of structure. The results compare different calculations carried out using these three formulations. A special case concerns the presence of mode veering in the Campbell diagram, which was studied in Ref. [13] in simplified gyroscopic systems.

\section{DYNAMIC MODELS OF THE ROTATING SOLID}

\subsection{General equation of motion}

In order to define the kinematics of the rotating solid, a non-rotating inertial frame of reference is defined (see Fig. 1). The third axis of the frame is the axis of revolution of the solid and has the 
direction of the angular velocity vector $\boldsymbol{\Omega}$. Let $\mathbf{u}$ be an Eulerian position vector that is associated with a spatial point of the undeformed configuration of the solid. The position vector $\mathbf{r}$ of a particle that is at $\mathbf{u}$ at time $t$ in the undeformed configuration can be formulated as follows

$$
\mathbf{r}=\mathbf{u}+\mathbf{w}(\mathbf{u}, t)
$$

where w contains the displacements associated with flexibility and the small rigid body displacements.

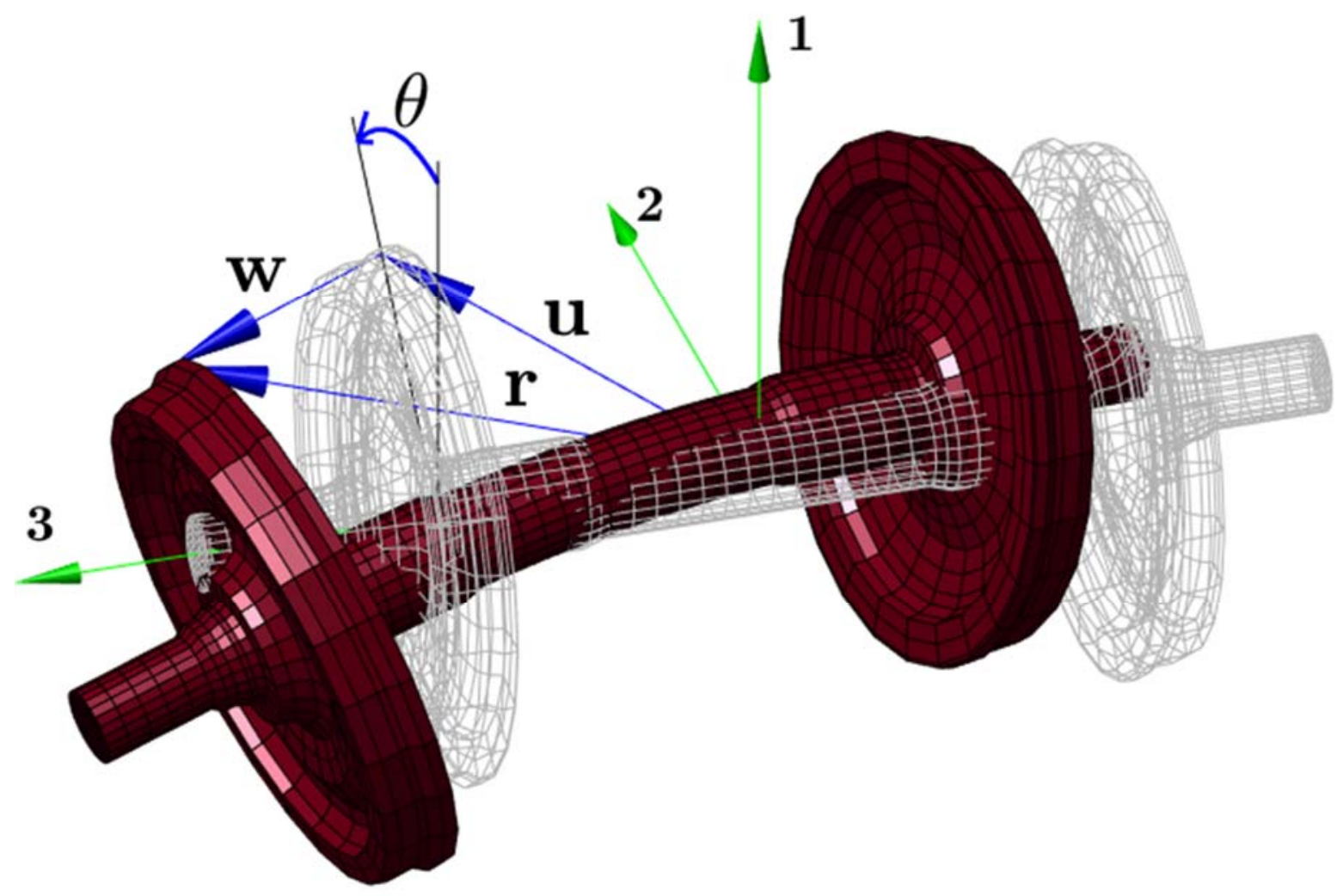

Fig. 1: Frame of reference and coordinates. In solid colour is represented a generic deformed configuration of the wheelset. The mesh corresponds to the undeformed configuration.

The velocity of the particle due to the rigid body rotation is

$$
\mathbf{v}=\left(\begin{array}{lll}
v_{1} & v_{2} & v_{3}
\end{array}\right)^{\mathrm{T}}=\Omega \mathbf{J} \mathbf{u}=\Omega \widetilde{\mathbf{u}},
$$

where $\Omega$ is the third component of the angular velocity vector $\boldsymbol{\Omega}$, 


$$
\mathbf{J}=\left(\begin{array}{ccc}
0 & -1 & 0 \\
1 & 0 & 0 \\
0 & 0 & 0
\end{array}\right)
$$

and $\widetilde{\mathbf{u}}=\left(\begin{array}{lll}-u_{2} & u_{1} & 0\end{array}\right)^{\mathrm{T}}$. Assuming that the angular velocity is constant, the velocity of the particle is computed through the material derivative of $\mathbf{r}$, and that is

$$
\frac{\mathrm{D} \mathbf{r}}{\mathrm{D} t}=\frac{\mathrm{Du}}{\mathrm{D} t}+\frac{\mathrm{D} \mathbf{w}}{\mathrm{D} t}=\sum_{i} v_{i} \frac{\partial \mathbf{u}}{\partial u_{i}}+\dot{\mathbf{w}}+\sum_{i} v_{i} \frac{\partial \mathbf{w}}{\partial u_{i}}=\Omega \mathbf{J} \mathbf{u}+\dot{\mathbf{w}}+\Omega \sum_{i} \widetilde{u}_{i} \frac{\partial \mathbf{w}}{\partial u_{i}}
$$

The kinetic energy of the solid is

$$
\begin{aligned}
T & =\frac{1}{2} \int_{V} \rho \frac{\mathrm{Dr}}{\mathrm{D} t} \frac{\mathrm{D} \mathbf{r}}{\mathrm{D} t} \mathrm{~d} v=\frac{1}{2} \Omega^{2} \int_{V} \rho \mathbf{u}^{\mathrm{T}} \widetilde{J} \mathbf{u} \mathrm{d} v+\frac{1}{2} \int_{V} \rho \dot{\mathbf{w}}^{\mathrm{T}} \dot{\mathbf{w}} \mathrm{d} v \\
& +\frac{1}{2} \Omega^{2} \int_{V} \rho\left(\sum_{i} \widetilde{u}_{i} \frac{\partial \mathbf{w}^{\mathrm{T}}}{\partial u_{i}}\right)\left(\sum_{i} \widetilde{u}_{i} \frac{\partial \mathbf{w}}{\partial u_{i}}\right) \mathrm{d} v+\Omega \int_{V} \rho \dot{\mathbf{w}}^{\mathrm{T}} \mathbf{J} \mathbf{u} \mathrm{d} v \\
& +\Omega^{2} \int_{V} \rho\left(\sum_{i} \widetilde{u}_{i} \frac{\partial \mathbf{w}^{\mathrm{T}}}{\partial u_{i}}\right) \mathbf{J} \mathbf{u} \mathrm{d} v+\Omega \int_{V} \rho \dot{\mathbf{w}}^{\mathrm{T}}\left(\sum_{i} \tilde{u}_{i} \frac{\partial \mathbf{w}}{\partial u_{i}}\right) \mathrm{d} v,
\end{aligned}
$$

where $V$ is the undeformed volume of the solid and the matrix $\widetilde{\mathbf{J}}$ is given by

$$
\widetilde{\mathbf{J}}=\mathbf{J}^{\mathrm{T}} \mathbf{J}=\left(\begin{array}{lll}
1 & 0 & 0 \\
0 & 1 & 0 \\
0 & 0 & 0
\end{array}\right)
$$

At this point, separation of variables is adopted and the displacements associated with the deformation are written as follows

$$
\mathbf{w}(\mathbf{u}, t)=\mathbf{S}(\mathbf{u}) \mathbf{q}(t),
$$

where $\mathbf{S}(\mathbf{u})$ is a set of basis functions and $\mathbf{q}(t)$ is the corresponding coordinate (or amplitude) vector. Through this approach, by differentiation of Eq. (5), the inertial terms of the EOM can be written as follows 


$$
\begin{aligned}
\frac{\mathrm{D}}{\mathrm{D} t}\left(\frac{\partial T}{\partial \dot{\mathbf{q}}}\right)^{\mathrm{T}}-\left(\frac{\partial T}{\partial \mathbf{q}}\right)^{\mathrm{T}} & =\int_{V} \rho \mathbf{S}^{\mathrm{T}} \mathbf{S} \mathrm{d} v \ddot{\mathbf{q}}+2 \Omega \int_{V} \rho \mathbf{S}^{\mathrm{T}}\left(-u_{2} \frac{\partial \mathbf{S}}{\partial u_{1}}+u_{1} \frac{\partial \mathbf{S}}{\partial u_{2}}\right) \mathrm{d} v \dot{\mathbf{q}} \\
& -\Omega^{2} \int_{V} \rho \mathbf{S}^{\mathrm{T}} \widetilde{\mathbf{J}} \mathbf{u} \mathrm{d} v+\Omega^{2} \int_{V} \rho \mathbf{S}^{\mathrm{T}}\left(u_{1} \frac{\partial \mathbf{S}}{\partial u_{1}}+u_{2} \frac{\partial \mathbf{S}}{\partial u_{2}}\right) \mathrm{d} v \mathbf{q} \\
& +\Omega^{2} \int_{V} \rho \mathbf{S}^{\mathrm{T}}\left(u_{2}^{2} \frac{\partial^{2} \mathbf{S}}{\partial u_{1}^{2}}-2 u_{1} u_{2} \frac{\partial^{2} \mathbf{S}}{\partial u_{1} \partial u_{2}}+u_{1}^{2} u_{1} \frac{\partial^{2} \mathbf{S}}{\partial u_{2}^{2}}\right) \mathrm{d} v \mathbf{q} .
\end{aligned}
$$

By performing a coordinate transformation from Cartesian to cylindrical coordinates, this yields

$$
\begin{aligned}
& u_{1}=r \cos \theta, \\
& u_{2}=r \sin \theta .
\end{aligned}
$$

Thus

$$
\begin{aligned}
& \frac{\partial}{\partial u_{1}}=\cos \theta \frac{\partial}{\partial r}-\frac{1}{r} \sin \theta \frac{\partial}{\partial \theta}, \\
& \frac{\partial}{\partial u_{2}}=\sin \theta \frac{\partial}{\partial r}+\frac{1}{r} \cos \theta \frac{\partial}{\partial \theta},
\end{aligned}
$$

and

$$
\begin{aligned}
& -u_{2} \frac{\partial}{\partial u_{1}}+u_{1} \frac{\partial}{\partial u_{2}}=\frac{\partial}{\partial \theta} \\
& u_{1} \frac{\partial}{\partial u_{1}}+u_{2} \frac{\partial}{\partial u_{2}}=r \frac{\partial}{\partial r} .
\end{aligned}
$$

By means of this coordinate change, Eq. (8) results in

$$
\begin{aligned}
\frac{\mathrm{D}}{\mathrm{D} t}\left(\frac{\partial T}{\partial \dot{\mathbf{q}}}\right)^{\mathrm{T}}-\left(\frac{\partial T}{\partial \mathbf{q}}\right)^{\mathrm{T}} & =\int_{V} \rho r \mathbf{S}^{\mathrm{T}} \mathbf{S} \mathrm{d} \vartheta \ddot{\mathbf{q}}+2 \Omega \int_{V} \rho r \mathbf{S}^{\mathrm{T}} \frac{\partial}{\partial \theta} \mathbf{S} \mathrm{d} \vartheta \dot{\mathbf{q}}-\Omega^{2} \int_{V} \rho r \mathbf{S}^{\mathrm{T}} \widetilde{\mathbf{J}} \mathbf{u} \mathrm{d} \vartheta \\
& +\Omega^{2} \int_{V} \rho r^{2} \mathbf{S}^{\mathrm{T}} \frac{\partial}{\partial r} \mathbf{S} \mathrm{d} \vartheta \mathbf{q}+\Omega^{2} \int_{V} \rho r \mathbf{S}^{\mathrm{T}}\left(\frac{\partial}{\partial \theta}\right)^{2} \mathbf{S} \mathrm{d} \vartheta \mathbf{q},
\end{aligned}
$$


where $\mathrm{d} \vartheta=\mathrm{d} \theta \mathrm{d} r \mathrm{~d} u_{3}$. The last term in Eq. (15) contains second-order derivatives, and this would produce convergence problems when the domain of integration is discretised into subdomains (e.g. finite elements). The basis $\mathbf{S}$ that will be adopted in this work uses quadratic shape functions for these subdomains, the continuity at its interfaces being $\mathrm{C}^{0}$. Consequently, the integration can only be evaluated properly if the maximum order of differentiation is 1 . Note that, as detailed in section 3.2, page 59 of Ref. [12] as convergence requirement, if the integrand has derivatives up to order $n$, then the interpolation has to guarantee that its $n-1$ derivatives are continuous ( $\mathrm{C}^{n-1}$ continuity). Therefore, the second-order derivative is unbounded in the last integral of Eq. (15). In order to reduce the order of the derivatives, the last term in Eq. (15) is integrated by parts, giving

$$
\int_{V} \rho r \mathbf{S}^{\mathrm{T}}\left(\frac{\partial}{\partial \theta}\right)^{2} \mathbf{S} \mathrm{d} \theta \mathrm{d} r \mathrm{~d} u_{3}=\int_{\partial V}\left[\rho r \mathbf{S}^{\mathrm{T}} \frac{\partial \mathbf{S}}{\partial \theta}\right]_{\theta=0}^{\theta=2 \pi} \mathrm{d} r \mathrm{~d} u_{3}-\int_{V} \rho r \frac{\partial \mathbf{S}^{\mathrm{T}}}{\partial \theta} \frac{\partial \mathbf{S}}{\partial \theta} \mathrm{d} \vartheta
$$

$\partial V$ being the external surface of the volume $V$. The integrand in the surface integral is zero and, consequently, the inertial terms of the EOM can be written as follows

$$
\begin{aligned}
\frac{\mathrm{D}}{\mathrm{D} t}\left(\frac{\partial T}{\partial \dot{\mathbf{q}}}\right)^{\mathrm{T}}-\left(\frac{\partial T}{\partial \mathbf{q}}\right)^{\mathrm{T}} & =\int_{V} \rho r \mathbf{S}^{\mathrm{T}} \mathbf{S} \mathrm{d} \vartheta \ddot{\mathbf{q}}+2 \Omega \int_{V} \rho r \mathbf{S}^{\mathrm{T}} \frac{\partial \mathbf{S}}{\partial \theta} \mathrm{d} \vartheta \dot{\mathbf{q}} \\
& +\Omega^{2} \int_{V} \rho r^{2} \mathbf{S}^{\mathrm{T}} \frac{\partial \mathbf{S}}{\partial r} \mathrm{~d} \vartheta \mathbf{q}-\Omega^{2} \int_{V} \rho r \frac{\partial \mathbf{S}^{\mathrm{T}}}{\partial \theta} \frac{\partial \mathbf{S}}{\partial \theta} \mathrm{d} \vartheta \mathbf{q}-\Omega^{2} \int_{V} \rho r \mathbf{S}^{\mathrm{T}} \widetilde{\mathbf{J}} \mathbf{u} \mathrm{d} \vartheta .
\end{aligned}
$$

\subsection{FE model through Eulerian coordinates (Model 1)}

Following the FEM, the coordinates that will be adopted in Eq. (7) are the Eulerian displacements $\mathbf{w}^{F E}$ at the nodes of the FE mesh, and the basis functions are the FE shape functions $\mathbf{N}$. By means of this approach, Eq. (7) can be rewritten as follows

$$
\mathbf{w}(\mathbf{u}, t)=\mathbf{N}(\mathbf{u}) \mathbf{w}^{F E}(t)
$$

Through this change, Eq. (17) results in

$$
\begin{aligned}
\frac{\mathrm{D}}{\mathrm{D} t}\left(\frac{\partial T}{\partial \dot{\mathbf{w}}^{F E}}\right)^{\mathrm{T}}-\left(\frac{\partial T}{\partial \mathbf{w}^{F E}}\right)^{\mathrm{T}} & =\int_{V} \rho r \mathbf{N}^{\mathrm{T}} \mathbf{N} \mathrm{d} \vartheta \ddot{\mathbf{w}}^{F E}+2 \Omega \int_{V} \rho r \mathbf{N}^{\mathrm{T}} \frac{\partial \mathbf{N}}{\partial \theta} \mathrm{d} \vartheta \dot{\mathbf{w}}^{F E} \\
& +\Omega^{2} \int_{V} \rho r^{2} \mathbf{N}^{\mathrm{T}} \frac{\partial \mathbf{N}}{\partial r} \mathrm{~d} \vartheta \mathbf{w}^{F E}-\Omega^{2} \int_{V} \rho r \frac{\partial \mathbf{N}^{\mathrm{T}}}{\partial \theta} \frac{\partial \mathbf{N}}{\partial \theta} \mathrm{d} \vartheta \mathbf{w}^{F E} \\
& -\Omega^{2} \int_{V} \rho r \mathbf{N}^{\mathrm{T}} \widetilde{\mathbf{J}} \mathbf{u} \vartheta .
\end{aligned}
$$


The FEM requires the discretisation of the domain in finite elements, so the integrals over the domain $V$ are separated in sums of the integrals over the volume of each finite element $V^{e}$, which gives

$$
\begin{aligned}
\frac{\mathrm{D}}{\mathrm{D} t}\left(\frac{\partial T}{\partial \dot{\mathbf{w}}^{F E}}\right)^{\mathrm{T}}-\left(\frac{\partial T}{\partial \mathbf{w}^{F E}}\right)^{\mathrm{T}} & =\sum_{e=1}^{E} \int_{V^{e}} \rho r \mathbf{N}^{\mathrm{T}} \mathbf{N} \mathrm{d} \vartheta \ddot{\mathbf{w}}^{F E}+2 \Omega \sum_{e=1}^{E} \int_{V^{e}} \rho r \mathbf{N}^{\mathrm{T}} \frac{\partial \mathbf{N}}{\partial \theta} \mathrm{d} \vartheta \dot{\mathbf{w}}^{F E} \\
& +\Omega^{2} \sum_{e=1}^{E} \int_{V^{e}} \rho r^{2} \mathbf{N}^{\mathrm{T}} \frac{\partial \mathbf{N}}{\partial r} \mathrm{~d} \vartheta \mathbf{w}^{F E}-\Omega^{2} \sum_{e=1}^{E} \int_{V^{e}} \rho r \frac{\partial \mathbf{N}^{\mathrm{T}}}{\partial \theta} \frac{\partial \mathbf{N}}{\partial \theta} \mathrm{d} \vartheta \mathbf{w}^{F E} \\
& -\Omega^{2} \sum_{e=1}^{E} \int_{V^{e}} \rho r \mathbf{N}^{\mathrm{T}} \tilde{\mathbf{J}} \mathbf{u} \mathrm{d} \vartheta
\end{aligned}
$$

where $E$ is the number of elements in the FE mesh. The matrices of the elements in this equation, which are

$$
\begin{gathered}
\mathbf{m}^{e}=\int_{V^{e}} \rho r \mathbf{N}^{\mathrm{T}} \mathbf{N} \mathrm{d} \vartheta \\
\mathbf{g}^{e}=\int_{V^{e}} \rho r \mathbf{N}^{\mathrm{T}} \frac{\partial \mathbf{N}}{\partial \theta} \mathrm{d} \vartheta, \\
\mathbf{b}_{1}^{e}=\int_{V^{e}} \rho r^{2} \mathbf{N}^{\mathrm{T}} \frac{\partial \mathbf{N}}{\partial r} \mathrm{~d} \vartheta, \\
\mathbf{b}_{2}^{e}=\int_{V^{e}} \rho r \frac{\partial \mathbf{N}^{\mathrm{T}}}{\partial \theta} \frac{\partial \mathbf{N}}{\partial \theta} \mathrm{d} \vartheta
\end{gathered}
$$

and

$$
\mathbf{c}^{e}=\int_{V^{e}} \rho r \mathbf{N}^{\mathrm{T}} \widetilde{\mathbf{J}} \mathbf{u} \mathrm{d} \vartheta
$$

are integrated through Gauss quadrature, and the resulting element matrices are assembled into global matrices following the standard FEM procedure. The matrix in Eq. (21) is the ordinary mass matrix in FEM.

\subsection{Eulerian modal coordinates (Model 2)}

Modal approaches are extensively adopted in vibration studies. The standard method adopts the mode shapes that are calculated with respect to a mobile reference frame that is associated with the nondeformed configuration of the solid. Since the undeformed solid has symmetry of revolution, in this 
work the mode shapes of the solid are adopted in this work as basis functions in a non-rotating frame of reference. Consequently, Eq. (7) becomes

$$
\mathbf{w}(\mathbf{u}, t)=\mathbf{\Phi}(\mathbf{u}) \mathbf{q}(t),
$$

with $\boldsymbol{\Phi}(\mathbf{u})$ being the modal matrix and $\mathbf{q}(t)$ the Eulerian modal coordinates. The modal matrix can be obtained through standard FE models, and it can be calculated as follows

$$
\boldsymbol{\Phi}(\mathbf{u})=\mathbf{N}(\mathbf{u}) \boldsymbol{\Phi}^{F E}
$$

where $\boldsymbol{\Phi}^{F E}$ is the matrix that contains the mode shape displacements at the nodes of the FE mesh. By means of this approach, the EOM becomes

$$
\begin{aligned}
\frac{\mathrm{D}}{\mathrm{D} t}\left(\frac{\partial T}{\partial \dot{\mathbf{q}}}\right)^{\mathrm{T}}-\left(\frac{\partial T}{\partial \mathbf{q}}\right)^{\mathrm{T}} & =\sum_{e=1}^{E} \boldsymbol{\Phi}^{F E^{\mathrm{T}}} \int_{V^{e}} \rho r \mathbf{N}^{\mathrm{T}} \mathbf{N} \mathrm{d} \vartheta \boldsymbol{\Phi}^{F E} \ddot{\mathbf{w}}^{F E}+2 \Omega \sum_{e=1}^{E} \boldsymbol{\Phi}^{F E^{\mathrm{T}}} \int_{V^{e}} \rho r \mathbf{N}^{\mathrm{T}} \frac{\partial \mathbf{N}}{\partial \theta} \mathrm{d} \vartheta \boldsymbol{\Phi}^{F E} \dot{\mathbf{w}}^{F E} \\
& +\Omega^{2} \sum_{e=1}^{E} \boldsymbol{\Phi}^{F E^{\mathrm{T}}} \int_{V^{e}} \rho r^{2} \mathbf{N}^{\mathrm{T}} \frac{\partial \mathbf{N}}{\partial r} \mathrm{~d} \vartheta \boldsymbol{\Phi}^{F E} \mathbf{w}^{F E} \\
& -\Omega^{2} \sum_{e=1}^{E} \boldsymbol{\Phi}^{F E^{\mathrm{T}}} \int_{V^{e}} \rho r \frac{\partial \mathbf{N}^{\mathrm{T}}}{\partial \theta} \frac{\partial \mathbf{N}}{\partial \theta} \mathrm{d} \vartheta \boldsymbol{\Phi}^{F E} \mathbf{w}^{F E}-\Omega^{2} \sum_{e=1}^{E} \boldsymbol{\Phi}^{F E^{\mathrm{T}}} \int_{V^{e}} \rho r \mathbf{N}^{\mathrm{T}} \widetilde{\mathbf{J}} \mathbf{u} \mathrm{d} \vartheta .
\end{aligned}
$$

The matrices of the elements in this equation, which are

$$
\begin{gathered}
\breve{\mathbf{m}}^{e}=\boldsymbol{\Phi}^{F E} \int_{V^{e}} \rho r \mathbf{N}^{\mathrm{T}} \mathbf{N} \mathrm{d} \vartheta \boldsymbol{\Phi}^{F E}, \\
\breve{\mathbf{g}}^{e}=\boldsymbol{\Phi}^{F E} \int_{V^{e}} \rho r \mathbf{N}^{\mathrm{T}} \frac{\partial \mathbf{N}}{\partial \theta} \mathrm{d} \vartheta \boldsymbol{\Phi}^{F E}, \\
\breve{\mathbf{b}}_{1}^{e}=\boldsymbol{\Phi}^{F E \mathrm{~T}} \int_{V^{e}} \rho r^{2} \mathbf{N}^{\mathrm{T}} \frac{\partial \mathbf{N}}{\partial r} \mathrm{~d} \vartheta \boldsymbol{\Phi}^{F E}, \\
\breve{\mathbf{b}}_{2}^{e}=\boldsymbol{\Phi}^{F E} \int_{V^{e}} \rho r^{2} \frac{\partial \mathbf{N}^{\mathrm{T}}}{\partial \theta} \frac{\partial \mathbf{N}}{\partial \theta} \mathrm{d} \vartheta \boldsymbol{\Phi}^{F E}
\end{gathered}
$$

and

$$
\breve{c}^{e}=\boldsymbol{\Phi}^{F E} \int_{V^{e}} \rho r \mathbf{N}^{\mathrm{T}} \tilde{\mathbf{J}} \mathbf{u} \mathrm{d} \vartheta
$$


The EOM matrices can be calculated following the same steps as in the preceding subsection (the matrices are obtained by pre- and post-multiplying Eqs. (21) to (25) by the modal matrix). Although the number of computations is slightly larger, the resulting EOM contains a reduced number of coordinates if a proper number of modes are considered.

\subsection{Eulerian axisymmetric model (Model 3)}

The displacements due to the deformations of the solid can be obtained by means of the following Fourier series

$$
\mathbf{w}\left(r, u_{3}, \theta\right)=\sum_{h=0}^{H} \widetilde{\mathbf{w}}_{h}\left(r, u_{3}\right) \cos \theta h+\sum_{h=1}^{H} \overline{\mathbf{w}}_{h}\left(r, u_{3}\right) \sin \theta h,
$$

where $H$ is the number of spatial harmonics, and $\widetilde{\mathbf{w}}_{h}$ and $\overline{\mathbf{w}}_{h}$ are the amplitudes of the harmonics (the index $h$ can be interpreted as the number of nodal diameters). The functions $\widetilde{\mathbf{w}}_{h}$ and $\overline{\mathbf{w}}_{h}$ can be formulated by means of the spatial harmonics at the nodes in a two-dimensional FE mesh of the solid section $\left(\widetilde{\mathbf{w}}_{h}^{F E}\right.$ and $\left.\overline{\mathbf{w}}_{h}^{F E}\right)$, as follows

$$
\begin{gathered}
\widetilde{\mathbf{w}}_{h}\left(r, u_{3}\right)=\mathbf{N}\left(r, u_{3}\right) \widetilde{\mathbf{w}}_{h}^{F E}, \\
\overline{\mathbf{w}}_{h}\left(r, u_{3}\right)=\mathbf{N}\left(r, u_{3}\right) \overline{\mathbf{w}}_{h}^{F E} .
\end{gathered}
$$

It must be highlighted that the shape functions that are adopted in the present subsection $\mathbf{N}\left(r, u_{3}\right)$ are associated with two-dimensional elements of the cross-section. The displacement vector is formulated as follows

$$
\mathbf{w}=\sum_{h=0}^{H} \mathbf{N} \widetilde{\mathbf{w}}_{h}^{F E} c_{h}+\sum_{n=1}^{H} \mathbf{N} \overline{\mathbf{w}}_{h}^{F E} S_{h}
$$

where

$$
\begin{gathered}
c_{h}=\cos \theta h, \\
s_{h}=\sin \theta h .
\end{gathered}
$$


The displacements can be rewritten as follows

$$
\mathbf{w}=\left[\begin{array}{llllll}
\mathbf{N} & \mathbf{N} c_{1} & \mathbf{N} s_{1} & \cdots & \mathbf{N} c_{H} & \mathbf{N} s_{H}
\end{array}\right]\left\{\begin{array}{c}
\widetilde{\mathbf{w}}_{0}^{F E} \\
\widetilde{\mathbf{w}}_{1}^{F E} \\
\overline{\mathbf{w}}_{1}^{F E} \\
\vdots \\
\widetilde{\mathbf{w}}_{H}^{F E} \\
\overline{\mathbf{w}}_{H}^{F E}
\end{array}\right\}
$$

where

$$
\mathbf{S}=\left[\begin{array}{llllll}
\mathbf{N} & \mathbf{N} c_{1} & \mathbf{N} s_{1} & \cdots & \mathbf{N} c_{H} & \mathbf{N} s_{H}
\end{array}\right]
$$

and

$$
\mathbf{q}=\left\{\begin{array}{c}
\widetilde{\mathbf{w}}_{0}^{F E} \\
\widetilde{\mathbf{w}}_{1}^{F E} \\
\overline{\mathbf{w}}_{1}^{F E} \\
\vdots \\
\widetilde{\mathbf{w}}_{H}^{F E} \\
\overline{\mathbf{w}}_{H}^{F E}
\end{array}\right\}
$$

Through this formulation, the general formulation in Eq. (17) that is based on Eq. (7) can be adopted. The first integral in the EOM in Eq. (17) is

$$
\mathbf{M}=\int_{V} \rho r \mathbf{S}^{\mathrm{T}} \mathbf{S} \mathrm{d} \theta \mathrm{d} r \mathrm{~d} u_{3}
$$

When performing the integral with respect to the angular coordinate $\theta$, all the cross terms are zero, resulting in

$$
\mathbf{M}=\left[\begin{array}{cccc}
2 \hat{\mathbf{m}} & \mathbf{0} & \cdots & \mathbf{0} \\
\mathbf{0} & \hat{\mathbf{m}} & \cdots & \mathbf{0} \\
\vdots & \vdots & \ddots & \vdots \\
\mathbf{0} & \mathbf{0} & \cdots & \hat{\mathbf{m}}
\end{array}\right]
$$

where $\mathbf{0}$ is the null matrix of appropriate dimensions (its dimensions are not indicated in this and the following equations for readability), and matrix $\hat{\mathbf{m}}$ is calculated as follows 


$$
\hat{\mathbf{m}}=\pi \int_{A} \rho r \mathbf{N}^{\mathrm{T}} \mathbf{N} \mathrm{d} r \mathrm{~d} u_{3}
$$

$A$ being the cross-section of the solid.

The second matrix in the EOM is

$$
\mathbf{G}=\int_{V} \rho r \mathbf{S}^{\mathrm{T}} \frac{\partial \mathbf{S}}{\partial \theta} \mathrm{d} \theta \mathrm{d} r \mathrm{~d} u_{3} .
$$

The derivative of the basis matrix $\mathbf{S}$ with respect to $\theta$ is

$$
\frac{\partial \mathbf{S}}{\partial \theta}=\left[\begin{array}{llllll}
\mathbf{0} & -\mathbf{N} s_{1} & \mathbf{N} c_{1} & \cdots & -\mathbf{N} H s_{H} & \mathbf{N} H c_{H}
\end{array}\right]
$$

By calculating the integrals with respect to $\theta$ in Eq. (46), all the blocks are zero except for the ones that couple the same-order sine and cosine harmonics, resulting in

$$
\mathbf{G}=\left[\begin{array}{cccccccc}
\mathbf{0} & \mathbf{0} & \mathbf{0} & \mathbf{0} & \mathbf{0} & \cdots & \mathbf{0} & \mathbf{0} \\
\mathbf{0} & \mathbf{0} & \hat{\mathbf{m}} & \mathbf{0} & \mathbf{0} & \cdots & \mathbf{0} & \mathbf{0} \\
\mathbf{0} & \hat{\mathbf{m}} & \mathbf{0} & \mathbf{0} & \mathbf{0} & \cdots & \mathbf{0} & \mathbf{0} \\
\mathbf{0} & \mathbf{0} & \mathbf{0} & \mathbf{0} & 2 \hat{\mathbf{m}} & \cdots & \mathbf{0} & \mathbf{0} \\
\mathbf{0} & \mathbf{0} & \mathbf{0} & -2 \hat{\mathbf{m}} & \mathbf{0} & \cdots & \mathbf{0} & \mathbf{0} \\
\vdots & \vdots & \vdots & \vdots & \vdots & \ddots & \vdots & \vdots \\
\mathbf{0} & \mathbf{0} & \mathbf{0} & \mathbf{0} & \mathbf{0} & \cdots & \mathbf{0} & H \hat{\mathbf{m}} \\
\mathbf{0} & \mathbf{0} & \mathbf{0} & \mathbf{0} & \mathbf{0} & \cdots & -H \hat{\mathbf{m}} & \mathbf{0}
\end{array}\right]
$$

Following the same methodology, the next matrices in the EOM are

$$
\mathbf{B}_{1}=\int_{V} \rho r^{2} \mathbf{S}^{\mathrm{T}} \frac{\partial \mathbf{S}}{\partial r} \mathrm{~d} \vartheta=\left[\begin{array}{cccc}
2 \hat{\mathbf{b}} & \mathbf{0} & \cdots & \mathbf{0} \\
\mathbf{0} & \hat{\mathbf{b}} & \cdots & \mathbf{0} \\
\vdots & \vdots & \ddots & \vdots \\
\mathbf{0} & \mathbf{0} & \cdots & \hat{\mathbf{b}}
\end{array}\right]
$$




$$
\mathbf{B}_{2}=\int_{V} \rho r \frac{\partial \mathbf{S}^{\mathrm{T}}}{\partial \theta} \frac{\partial \mathbf{S}}{\partial \theta} \mathrm{d} \vartheta=\left[\begin{array}{cccccccc}
\mathbf{0} & \mathbf{0} & \mathbf{0} & \mathbf{0} & \mathbf{0} & \cdots & \mathbf{0} & \mathbf{0} \\
\mathbf{0} & \hat{\mathbf{m}} & \mathbf{0} & \mathbf{0} & \mathbf{0} & \cdots & \mathbf{0} & \mathbf{0} \\
\mathbf{0} & \mathbf{0} & \hat{\mathbf{m}} & \mathbf{0} & \mathbf{0} & \cdots & \mathbf{0} & \mathbf{0} \\
\mathbf{0} & \mathbf{0} & \mathbf{0} & 2^{2} \hat{\mathbf{m}} & \mathbf{0} & \cdots & \mathbf{0} & \mathbf{0} \\
\mathbf{0} & \mathbf{0} & \mathbf{0} & \mathbf{0} & 2^{2} \hat{\mathbf{m}} & \cdots & \mathbf{0} & \mathbf{0} \\
\vdots & \vdots & \vdots & \vdots & \vdots & \ddots & \vdots & \vdots \\
\mathbf{0} & \mathbf{0} & \mathbf{0} & \mathbf{0} & \mathbf{0} & \cdots & H^{2} \hat{\mathbf{m}} & \mathbf{0} \\
\mathbf{0} & \mathbf{0} & \mathbf{0} & \mathbf{0} & \mathbf{0} & \cdots & \mathbf{0} & H^{2} \hat{\mathbf{m}}
\end{array}\right],
$$

and

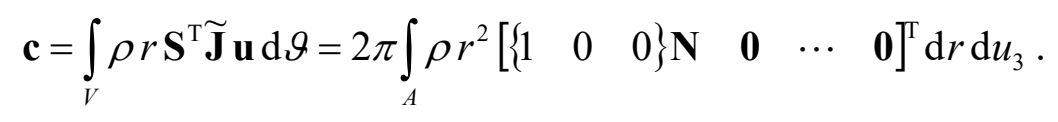

where matrix $\hat{\mathbf{b}}$ is

$$
\hat{\mathbf{b}}=\pi \int_{A} \rho r^{2} \mathbf{N}^{\mathrm{T}} \frac{\partial \mathbf{N}}{\partial r} \mathrm{~d} r \mathrm{~d} u_{3} .
$$

The integrals in Eqs. (45), (51) and (52) are used to develop the EOM, following the procedure that was carried out in the preceding subsections.

\subsection{Equation of motion}

Whichever approach is adopted, the resulting EOM for the rotating solid can be written in a matrix form as follows

$$
\mathbf{M} \ddot{\mathbf{q}}+2 \Omega \mathbf{G} \dot{\mathbf{q}}+\left(\mathbf{K}+\Omega^{2}\left(\mathbf{B}_{1}-\mathbf{B}_{2}\right)\right) \mathbf{q}=\Omega^{2} \mathbf{c}+\mathbf{Q}
$$

where $\mathbf{Q}$ is the generalised force vector, $\mathbf{G}$ is the skew-symmetric gyroscopic matrix, $\mathbf{B}_{1}$ and $\mathbf{B}_{2}$ are the stiffening matrices, and the vector $\mathbf{c}$ is a constant generalised-force vector that is responsible for the centrifugal "forces". Bearing in mind that the elastic energy expression does not depend on whether the standard Lagrangian or the Eulerian approach is adopted, the matrix $\mathbf{K}$ is the stiffness matrix, which is either the standard one in FEM, or the diagonal matrix containing the squared natural frequencies in the modal approach. The resulting EOM is linear since the angular velocity of the solid is supposed to be constant (which is plausible for a railway wheelset) and the constitutive relationship is linear. 
The dimensions of Eq. (53) depend on the model. Model 1 has the same dimensions as a standard FEM; the dimensions for Model 2 are the number of vibration modes that have been considered; and for Model 3 it is the number of degrees of freedom of the two-dimensional FE mesh times $H$ (the number of harmonics).

The influence of the rotation on the results can be explained mathematically by the terms in the EOM that contain $\Omega$. The gyroscopic term contains the product of the generalised-velocity vector and $\Omega$ . In addition there are two terms that contain the product of $\Omega^{2}$ and the generalised-displacement vector, the sum of which produces the total stiffening matrix due to rotation.

Since the formulation of the generalised force $\mathbf{Q}$ has to satisfy that the virtual work associated with the external forces is $\delta W=\mathbf{Q}^{\mathrm{T}} \delta \mathbf{q}$, which only depends on displacements, the generalised-force expression is the same as for the corresponding Lagrangian approach.

When adopting the axisymmetric model, the block matrices that couple the different harmonics (number of nodal diameters) are zero, as can be seen in Eqs. (44), and (48) to (50). Consequently, Eq. (53) can be analysed for each harmonic separately. The EOM for the $h$-th harmonic when $h>0$ is

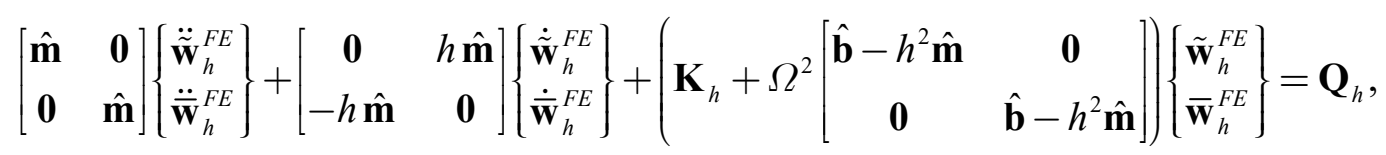

and the corresponding EOM when $h=0$ is

$$
2 \hat{\mathbf{m}} \ddot{\tilde{\mathbf{w}}}_{0}^{F E}+\left(\mathbf{K}_{0}+2 \Omega^{2} \hat{\mathbf{b}}\right) \tilde{\mathbf{w}}_{0}^{F E}=2 \Omega^{2} \pi \int_{A} \rho r^{2} \mathbf{N}^{\mathrm{T}}\left\{\begin{array}{l}
1 \\
0 \\
0
\end{array}\right\} \mathrm{d} r \mathrm{~d} u_{3}+\mathbf{Q}_{0}
$$

$\mathbf{Q}_{h}$ and $\mathbf{K}_{h}$ being the generalised force vector and the stiffness matrix associated with the $h$-th harmonic.

The formulation obtained in this work is adequate to calculate the dynamic coupling between the rotating solid (the wheelset) and a non-rotating structure (the railway track). Eq. (53) is linear with constant matrices, which is not an exclusive advantage of Eulerian methods (see an example in Ref. [14], where a total Lagrangian approach is adopted for tire modelling). As will be studied in the following section, the EOM also allows to solve the eigenvalue problem, which provides the equivalent natural frequencies and mode shapes of the rotating solid. 


\section{RESULTS}

The present section presents some results that allow the different approaches to be compared. Additionally, some effects of rotation are shown that can be simulated with the approaches presented in the preceding section. The basis of this study is a railway wheel, which is considered boundaryfree in all the calculations. The wheel diameter is $853 \mathrm{~mm}$.

\subsection{Modes and mode coupling}

Two FE models are adopted. The first is a two-dimensional mesh that is shown in Fig. 2, which is used for Model 3. The mesh is formed of 4-node quadrilateral elements and has 1026 degrees of freedom (DOF). The second is a three-dimensional (3-D) mesh of the wheel, meshed with 8-node brick elements (135432 DOF), which is obtained through angular extrusion of the two-dimensional mesh. This is shown in Fig. 3(a) and is used for Models 1 and 2. Fig. 3 also presents examples of the non-rotating mode shapes; these are associated with natural frequencies between 2900 and $3300 \mathrm{~Hz}$, which will be analysed below. These are examples of modes with 0, 2, 3 (two cases) and 5 nodal diameters. The calculation of the modal properties is approximately 30000 times faster when the axisymmetric model is adopted than with the 3-D model. The differences between the non-rotating natural frequencies from the axisymmetric and 3-D models are less than $1 \%$ for all modes below 5 $\mathrm{kHz}$.

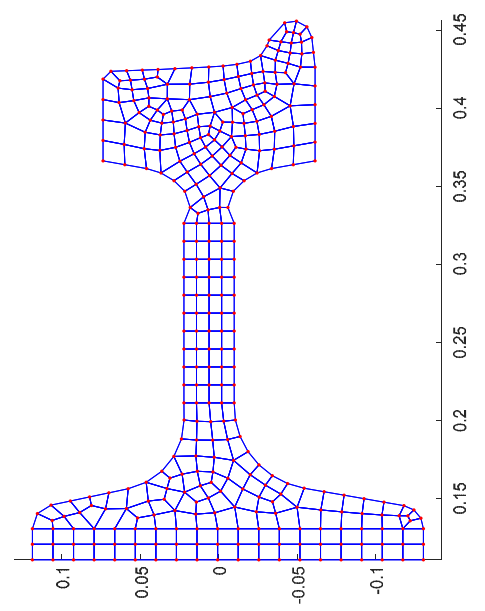

Fig. 2: Two-dimensional FE mesh of the wheel section.

Fig. 4 represents a map of the magnitudes of the terms in the gyroscopic matrix and the total stiffening matrix, when only the modes in Fig. 3 are considered. The calculation is done by means of the model 
based on Eulerian modal coordinates (Model 2). The figure shows the values of the $9 \times 9$ matrices, which are associated with one mode of multiplicity 1 (i.e. with 0 nodal diameters, plotted in Fig. 3(e)) and four modes with multiplicity 2 (modes b, c, d and $\mathrm{f}$ in Fig. 3). The rows and columns have been marked with letters ( $b$ to $f$ ) referring to the notation of the modes in Fig. 3. From this result the following conclusions can be deduced:

- There is strong coupling between the modes with the same number of nodal diameters. E.g., the maps show coupling between modes $\mathrm{b}$ and $\mathrm{f}$ in Fig. 3, which both have 3 nodal diameters.

- Eq. (48) presents skew-symmetric components in the gyroscopic matrix that can be seen in the map of Fig. 4(a).

- Eqs. (49) and (50) present self-coupling terms that are found as diagonal terms in Fig. 4(b).

- The gyroscopic matrix in Eq. (48) has zero components for modes with zero nodal diameters. The diagonal component associated with mode e is zero (or close to zero) in Fig. 4(a).
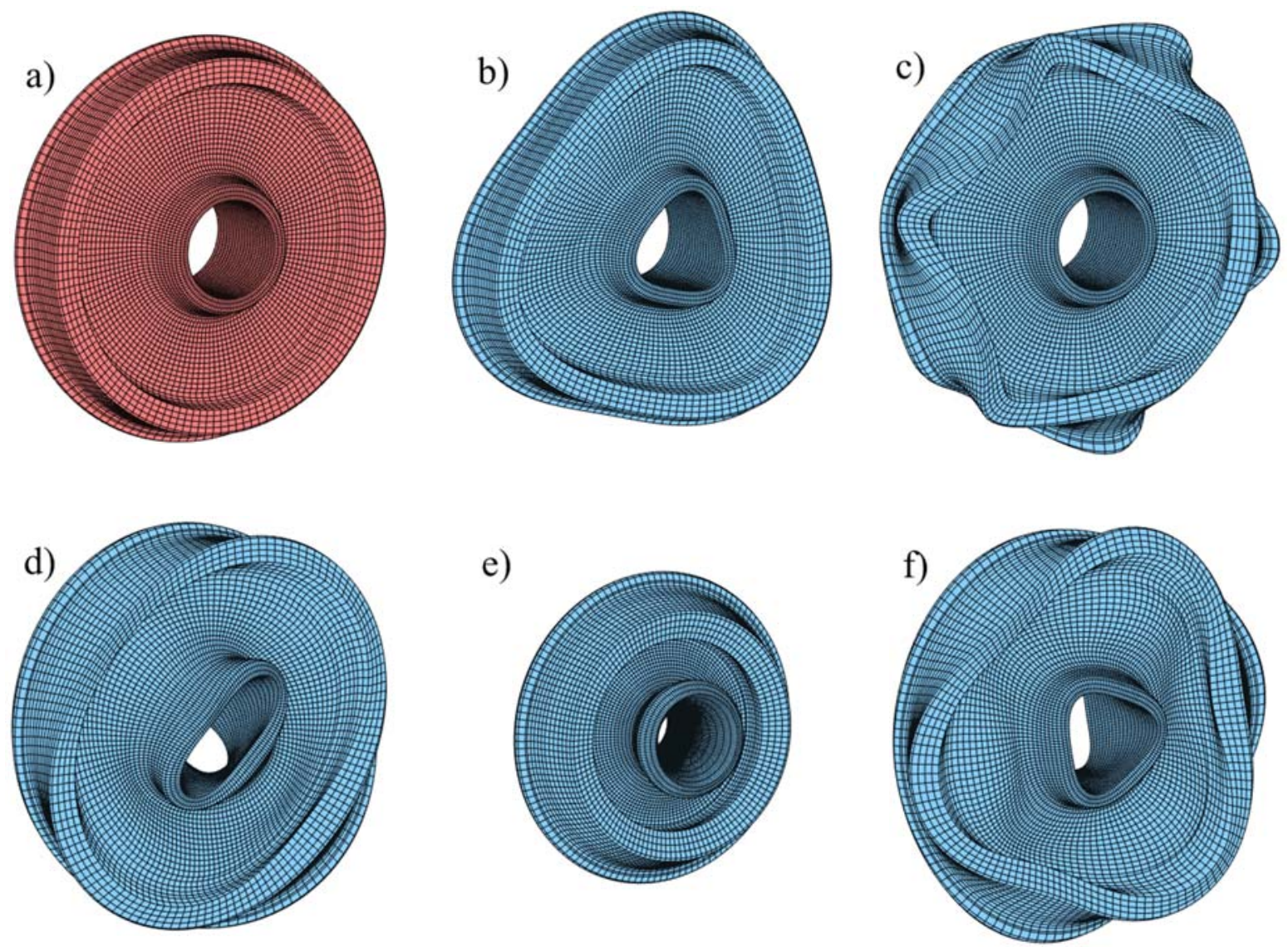

Fig. 3: Three-dimensional FE mesh of the wheel and five examples of non-rotating mode shapes:

a) Undeformed FE mesh; b) $2950 \mathrm{~Hz}, 3$ nodal diameters, radial mode; c) $3084 \mathrm{~Hz}, 5 \mathrm{nodal}$ diameters, 0 nodal circles; d) $3142 \mathrm{~Hz}, 2$ nodal diameters, 1 nodal circle; e) $3218 \mathrm{~Hz}, 0$ nodal diameters, radial mode; f) $3286 \mathrm{~Hz}, 3$ nodal diameters, 1 nodal circle. 
These conclusions are in line with the axisymmetric model. There are, however, differences with the axisymmetric model such as the small components of the matrices that couple modes with different numbers of nodal diameters.

a)

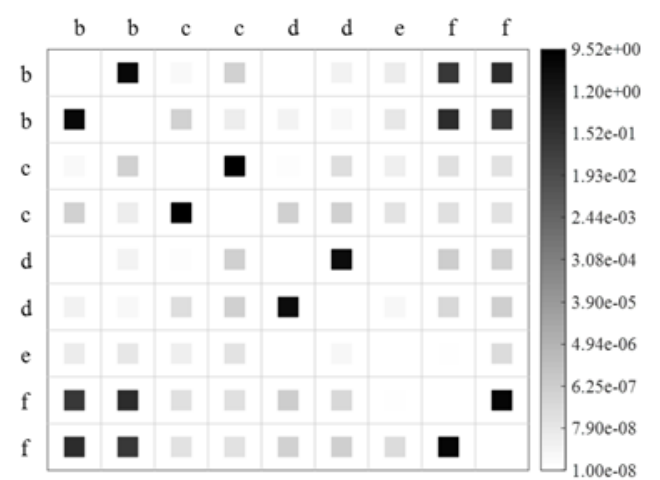

b)

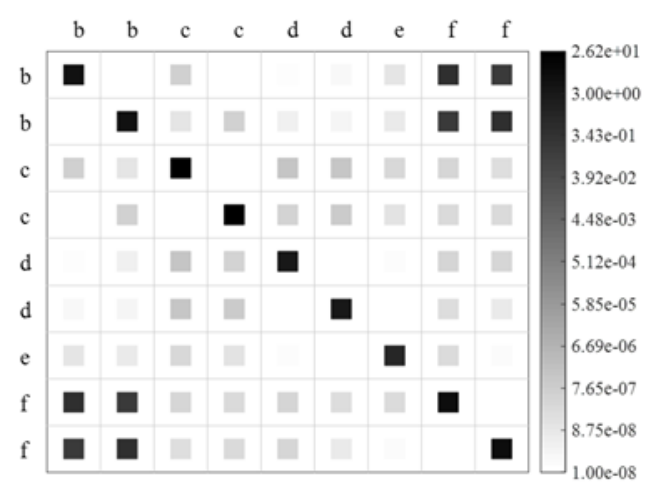

Fig. 4: Map of the gyroscopic (a) and the stiffening (b) matrices, calculated by means of the Eulerian modal model when only the modes in Fig. 3 are considered. The greyscale is in logarithmic scale.

\subsection{Influence of rotation on the modal properties}

Fig. 5 presents the Campbell diagram, which shows the natural frequencies of the rotating solid versus the angular velocity of the solid (in the present case, the speed of the vehicle that produces such angular velocity). The calculations compare results from the Eulerian modal coordinate model and the axisymmetric model. The agreement is good in general, and the differences must be associated with the FE discretisation error and the simplification adopted in Eq. (34) in the axisymmetric model (where the influence of the angular coordinate is separated from the other coordinates). It must be pointed out that the discretisation error affects the different models in a different manner; as an obvious example, the number of elements in the circumferential direction just affects the threedimensional model, and can be observed when the pair of modes with multiplicity 2 at zero angular velocity are calculated at different frequencies using the 3-D model (there is a significant example at $4150 \mathrm{~Hz}$, where natural frequencies are calculated at 4130 and $4152 \mathrm{~Hz}$ ). Regarding the computational time, both modal and axisymmetric models have similar computational cost (the figure can be generated in a few seconds) whereas the calculation of the full 3-D rotating FE model cannot be achieved by using a Matlab program on a conventional personal computer.

From Ref [15], the natural frequencies of the rotating wheelset can be approximated from the nonrotating ones $\omega_{n}$ by means of the following formulae 


$$
\begin{aligned}
& \omega_{n b}=\omega_{n}-h \Omega, \\
& \omega_{n f}=\omega_{n}+h \Omega,
\end{aligned}
$$

$\omega_{n b}$ being the frequency of the backward mode (which rotates in the opposite direction to the angular velocity), and $\omega_{n f}$ the frequency of the forward mode (which rotates in the same direction). Table 1 presents the natural frequencies of the rotating and non-rotating wheel; the former have been calculated through the axisymmetric model, and Eqs. (56,57). The columns marked 'Difference for backward/forward' indicate the difference between the FE result and Eqs $(56,57)$.

\begin{tabular}{|c|c|c|c|c|c|c|c|}
\hline $\begin{array}{c}\text { Natural } \\
\text { frequency } \\
\omega_{n} \\
{[\mathrm{~Hz}]}\end{array}$ & $\begin{array}{c}\text { Number of } \\
\text { nodal } \\
\text { diameters } \\
h \\
\end{array}$ & $\begin{array}{c}\text { Backward } \\
\text { frequency at } \\
300 \mathrm{~km} / \mathrm{h} \\
\omega_{n b} \\
{[\mathrm{~Hz}]}\end{array}$ & $\begin{array}{c}\text { Backward } \\
\text { formula } \\
\omega_{n}-h \Omega \\
{[\mathrm{Hz}]}\end{array}$ & $\begin{array}{c}\text { Difference } \\
\text { for } \\
\text { backward } \\
{[\%]}\end{array}$ & $\begin{array}{c}\text { Forward } \\
\text { frequency at } \\
300 \mathrm{~km} / \mathrm{h} \\
{[\mathrm{Hz}]} \\
\omega_{n f}\end{array}$ & $\begin{array}{c}\text { Forward } \\
\text { formula } \\
\omega_{n}+h \Omega \\
{[\mathrm{Hz}]}\end{array}$ & $\begin{array}{c}\text { Difference } \\
\text { for forward } \\
{[\%]}\end{array}$ \\
\hline 449.99 & 2 & 393.54 & 387.79 & 1.46 & 517.93 & 512.18 & 1.11 \\
\hline 952.36 & 1 & 923.25 & 921.26 & 0.22 & 985.44 & 983.46 & 0.20 \\
\hline 1158.09 & 3 & 1067.47 & 1064.80 & 0.25 & 1254.06 & 1251.38 & 0.21 \\
\hline 1722.92 & 2 & 1660.37 & 1660.73 & 0.02 & 1784.76 & 1785.12 & 0.02 \\
\hline 2065.55 & 4 & 1942.80 & 1941.16 & 0.08 & 2191.58 & 2189.94 & 0.07 \\
\hline 2116.03 & 2 & 2053.89 & 2053.83 & 0.00 & 2178.29 & 2178.22 & 0.00 \\
\hline 2435.29 & 1 & 2407.18 & 2404.19 & 0.12 & 2469.38 & 2466.38 & 0.12 \\
\hline 2747.87 & 1 & 2721.25 & 2716.77 & 0.16 & 2783.44 & 2778.97 & 0.16 \\
\hline 2950.14 & 3 & 2856.78 & 2856.85 & 0.00 & 3043.36 & 3043.43 & 0.00 \\
\hline 3078.71 & 5 & 2924.40 & 2923.22 & 0.04 & 3235.37 & 3234.19 & 0.04 \\
\hline 3158.75 & 2 & 3096.43 & 3096.56 & 0.00 & 3218.79 & 3220.95 & 0.07 \\
\hline 3296.85 & 3 & 3235.37 & 3203.56 & 0.98 & 3389.97 & 3390.14 & 0.01 \\
\hline 3697.72 & 2 & 3635.20 & 3635.52 & 0.01 & 3759.59 & 3759.91 & 0.01 \\
\hline 3760.97 & 4 & 3636.56 & 3636.58 & 0.00 & 3885.34 & 3885.36 & 0.00 \\
\hline 4141.57 & 4 & 4016.99 & 4017.18 & 0.00 & 4265.77 & 4265.96 & 0.00 \\
\hline 4455.68 & 1 & 4433.90 & 4424.58 & 0.21 & 4496.10 & 4486.78 & 0.21 \\
\hline 4584.77 & 5 & 4429.31 & 4429.28 & 0.00 & 4740.28 & 4740.26 & 0.00 \\
\hline 4895.91 & 3 & 4802.40 & 4802.61 & 0.00 & 4988.98 & 4989.20 & 0.00 \\
\hline
\end{tabular}

Table 1: Natural frequencies of the wheel below $5 \mathrm{kHz}$ of modes with 1 or more nodal diameters. 


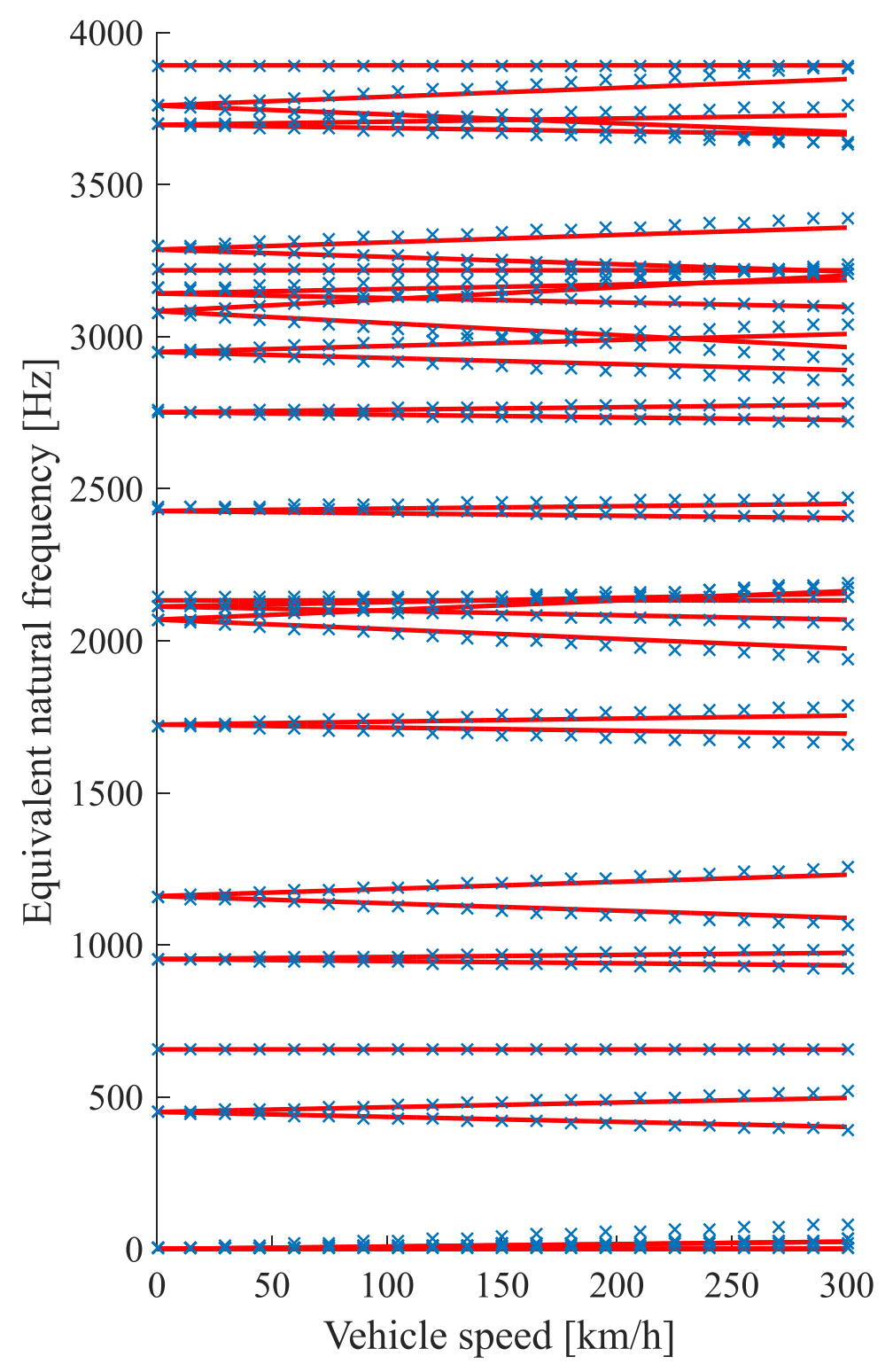

Fig. 5: Campbell diagram of the wheel. The abscissa shows the speed of the vehicle that produces the corresponding angular velocity of the wheel. The blue crosses correspond with calculations carried out with the axisymmetric model (Model 3). The red solid lines were calculated with the Eulerian modal coordinates (Model 2).

Fig. 6 plots a section of the Campbell diagram calculated by means of the model that adopts Eulerian modal coordinates with only the modes shown in Fig. 3 implemented. The modal assurance criterion (MAC, see Ref. [16]) is used in this calculation in order to relate the modes that are calculated at different angular speeds with each other. The modes that, according to the MAC index, are found to be the same, are plotted in the same colour and line style in this figure. 
The variation of the natural frequencies of the rotating solid with the angular velocity may cause two natural frequencies to be coincident, which can be detected in the Campbell diagram through the apparent intersection of two lines associated with different modes. According to Ref. [17], the crossing point in the Campbell diagram has important consequences in being responsible for rail corrugation growth in curves. Through simulation, in that reference it is shown that when implementing the conditions of curve negotiation, periodic wear occurs on the inner rail of the track if two natural frequencies in the Campbell diagram are approximately coincident. However, a more detailed analysis of the Campbell diagram shows that the crossing points are actually mode veering. The mode veering has been described for simplified gyroscopic models in [13]. The details of this behaviour can be assessed by means of the results in Figs 7 and 8.

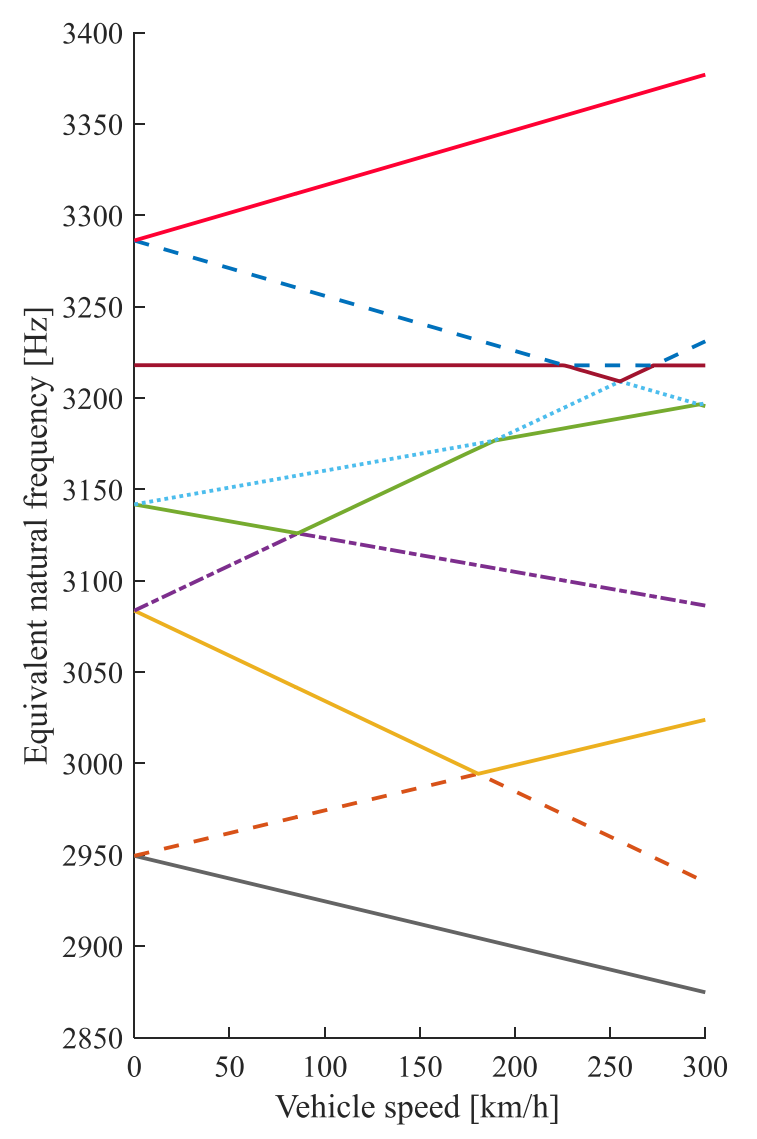

Fig. 6: Campbell diagram of the wheel when only the modes plotted in Fig. 3 are considered.

Fig. 7 corresponds to a zoomed view of Fig. 6. The figure shows the veering associated with modes $\mathrm{c}$ and $\mathrm{d}$ (as in the previous case, the notation $\mathrm{c}$ and $\mathrm{d}$ refers to Fig. 3). Instead of crossing, the modes 
change rapidly, with mode c changing into mode $\mathrm{d}$, and vice versa. Nevertheless it should be noted that the range of speeds over which this phenomenon occurs is very small.

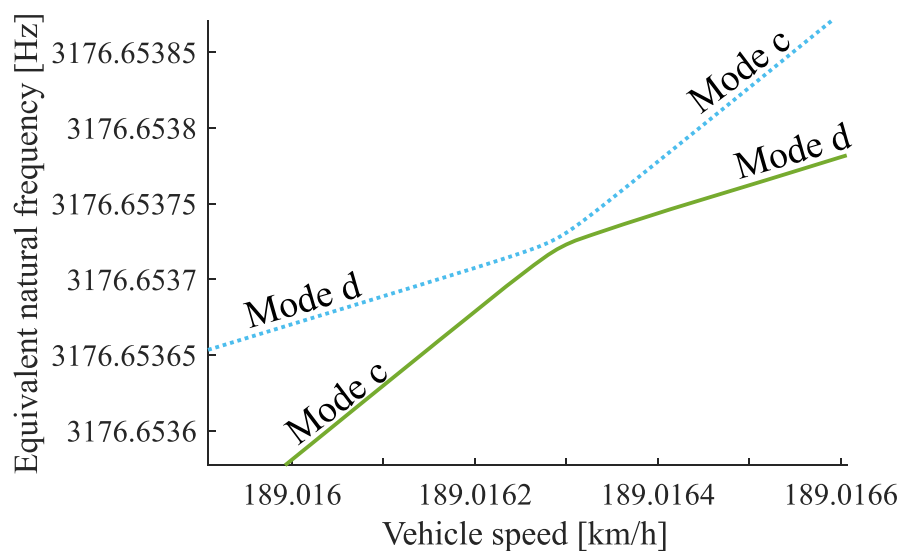

Fig. 7: Zoomed view of the veering zone in the Campbell diagram shown in Fig. 6.
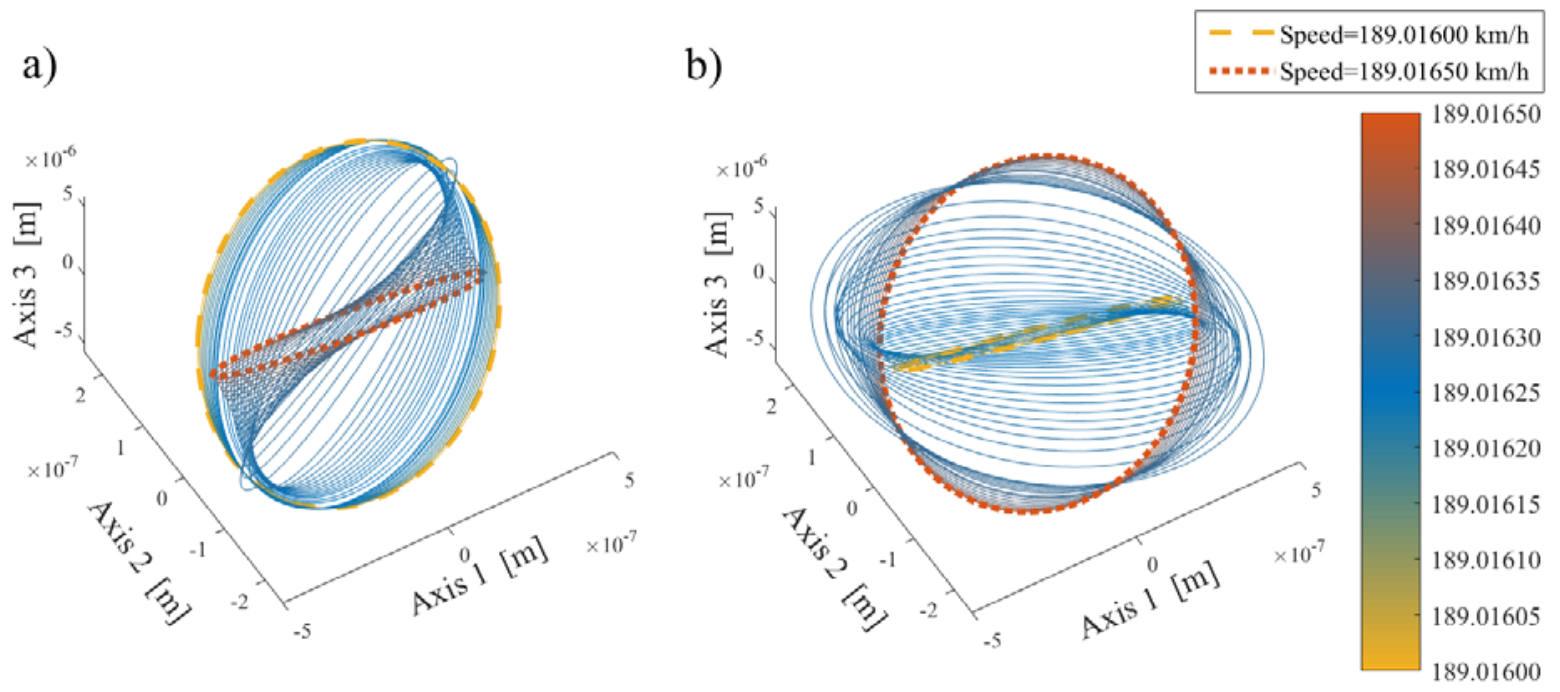

Fig. 8: Modal orbits that are calculated for the speed range where the veering takes place. a) Mode plotted in continuous green trace in Fig. 7. b) Mode plotted in dashed blue trace in Fig. 7.

Fig. 8 presents different modal orbits for a point located on the wheel tread at $70 \mathrm{~mm}$ from the flangeback. These trajectories are ellipses showing how the vibration varies during a cycle; they have been calculated for a set of vehicle speeds and they are plotted in different colours, according to the colour 
bar of the figure. Fig. 8(a) presents the orbits associated with the natural frequency that is plotted as a solid green line in Fig. 7, whereas the corresponding orbits of the natural frequency plotted as a dashed blue line are shown in Fig. 8(b). This result shows how the modes veer when the vehicle speed changes by just $0.0005 \mathrm{~km} / \mathrm{h}$. Modes $\mathrm{c}$ and $\mathrm{d}$ have different numbers of nodal diameters and consequently this behaviour cannot be predicted by means of the axisymmetric model.

\section{CONCLUSIONS}

Three closed-form formulations are presented for the study of the dynamics of rotating solids with axisymmetric geometry. The methodology is based on a general equation of motion in which the displacements due to the small deformations are written by means of a basis that adopts an Eulerian approach. The resulting Eulerian coordinates reduce the computational cost when the solid interacts with a non-rotating structure, and enable the natural frequencies and the frequency response functions of the rotating solid to be obtained. The general formulation in cylindrical coordinates is compact and avoids numerical issues since it only contains first-order derivatives with respect to the coordinates. Three different sets of basis functions (FEM shape functions, undamped mode shapes and axisymmetric FE shape functions) are adopted, producing three formulations. The formulation based on FEM generates a very large number of degrees of freedom, but its modelling cost is small since it follows the general FEM procedure for building the equations of motion. The models that adopt Eulerian modal coordinates and axisymmetric coordinates have similar (and reduced) computational costs. The Eulerian modal approach allows the weak coupling to be calculated that produces the mode veering, which has been associated in the literature with the wavelength fixing mechanism in certain rail corrugation problems. The axisymmetric model can in general reduce the discretisation problems occurring when a coarse mesh is adopted, and it avoids the need for a complex three-dimensional FE model with a high number of degrees of freedom.

\section{ACKNOWLEDGEMENTS}

The authors gratefully acknowledge the financial support of the European Commission through the project "RUN2Rail - Innovative RUNning gear soluTiOns for new dependable, sustainable, intelligent and comfortable RAIL vehicles" (Horizon 2020 Shift2Rail JU call 2017, grant number 777564), as well as Ministerio de Ciencia, Innovación y Universidades and the European Regional Development Fund (projects TRA2017-84701-R and MTM2015-64013-P). 


\section{REFERENCES}

[1] C.W. Lee, Vibration analysis of rotors, Kluwer, Dordrecht, 1993.

[2] G. Genta, Dynamics of rotating systems, Springer, New York, 2005.

[3] M.A. Brown, A.A. Shabana, Application of multibody methodology to rotating shaft problems, Journal of Sound and Vibration 204 (1997) 439-457.

[4] A.A. Shabana, Dynamics of Multibody Systems, Cambridge University Press, Cambridge, 2005.

[5] L.W. Chen, D.M. Ku, Analysis of whirl speeds of rotor-bearing systems with internal damping by $\mathrm{C}^{0}$ finite elements, Finite Elements in Analysis and Design 9 (1991) 169-176.

[6] G. Jacquet-Richardet, G. Ferraris, P. Rieutord, Frequencies and modes of rotating flexible bladed disc-shaft assemblies: a global cyclic symmetry approach, Journal of Sound and Vibration 191 (1996) 901-915.

[7] U. Nackenhorst, The ALE-formulation of bodies in rolling contact: theoretical foundations and finite element approach, Computer Methods in Applied Mechanics and Engineering 193 (39-41) (2004) 4299-4322.

[8] T.J.R. Hughes, W.K. Liu, T.K. Zimmermann, Lagrangian-Eulerian finite element formulation for incompressible viscous flows, Computer Methods in Applied Mechanics and Engineering 29 (1981) $329-349$.

[9] I. López Arteaga, R.E.A. Blom, N.B. Roozen, H. Nijmeijer, Modelling vibrations on deformed rolling tyres - a modal approach, Journal of Sound and Vibration 307 (2007) 481-494.

[10] J. Fayos, L. Baeza, F.D. Denia, J.E. Tarancón, An Eulerian coordinate-based method for analysing the structural vibrations of a solid of revolution rotating about its main axis, Journal of Sound and Vibration 306 (2007) 618-635.

[11] J. Martínez-Casas, L. Mazzola, L. Baeza, S. Bruni, Numerical estimation of stresses in railway axles using a train-track interaction model, International Journal of Fatigue 47 (2013) 18-30.

[12] O.C. Zienkiewicz, R.L. Taylor, J.Z. Zhu, The finite element method: Its basis and fundamentals, Elsevier Butterworth-Heinemann, Oxford, 2005. 
[13] S. Vidoli, F. Vestroni, Veering Phenomena in Systems With Gyroscopic Coupling, Journal of Applied Mechanics 72(5) (2005) 641-647.

[14] J.R. Cho, K.W.Kim, D.H. Jeon, W.S.Yoo, Transient dynamic response analysis of 3-D patterned tire rolling over cleat, European Journal of Mechanics - A/Solids 24 (2005) 519-531.

[15] D.J. Thompson. Wheel-rail noise: theoretical modelling of the generation of vibrations. PhD thesis, University of Southampton, 1990.

[16] N.M.M. Maia, J.M.M. Silva (ED.), Theoretical and Experimental Modal Analysis, Research Studies Press, Baldock, 1997.

[17] P. Vila, L. Baeza, J. Martínez-Casas, J. Carballeira, Rail corrugation growth accounting for the flexibility and rotation of the wheelset and the non-Hertzian and non-steady state effects at contact patch, Vehicle System Dynamics 52 (Supplement 1) (2014) 92-108. 